\title{
A-to-I-edited miRNA-379-5p inhibits cancer cell proliferation through CD97-induced apoptosis
}

\author{
Xiaoyan Xu, ${ }^{1,2}$ Yumeng Wang, ${ }^{2,3}$ Kamalika Mojumdar, ${ }^{2}$ Zhicheng Zhou, ${ }^{2,4}$ Kang Jin Jeong, ${ }^{4}$ Lingegowda S. Mangala, ${ }^{5,6}$ \\ Shuangxing Yu, ${ }^{4}$ Yiu Huen Tsang, ${ }^{7}$ Cristian Rodriguez-Aguayo, ${ }^{8}$ Yiling Lu, ${ }^{4}$ Gabriel Lopez-Berestein, ${ }^{6,8}$ Anil K. Sood, ${ }^{5,6}$ \\ Gordon B. Mills, ${ }^{4}$ and Han Liang ${ }^{2,3,4}$
}

\begin{abstract}
'Department of Pathophysiology, College of Basic Medical Science, China Medical University, Shenyang, Liaoning Province, China. ${ }^{2}$ Department of Bioinformatics and Computational Biology, The University of Texas MD Anderson Cancer Center, Houston, Texas, USA. ${ }^{3}$ Graduate Program in Quantitative and Computational Biosciences, Baylor College of Medicine, Houston, Texas, USA. ${ }^{4}$ Department of Systems Biology, ${ }^{5}$ Department of Gynecologic Oncology and Reproductive Medicine, and ${ }^{6}$ Center for RNA Interference and Non-Coding RNAs, The University of Texas MD Anderson Cancer Center, Houston, Texas, USA. 'Department of Molecular and Human Cenetics, Baylor College of Medicine, Houston, Texas, USA. ${ }^{8}$ Department of Experimental Therapeutics, The University of Texas MD Anderson Cancer Center, Houston, Texas, USA.
\end{abstract}

\begin{abstract}
Both miRNAs and A-to-I RNA editing, a widespread nucleotide modification mechanism, have recently emerged as key players in cancer pathophysiology. However, the functional impact of RNA editing of miRNAs in cancer remains largely unexplored. Here, we focused on an ADAR2-catalyzed RNA editing site within the miR-379-5p seed region. This site was under-edited in tumors relative to normal tissues, with a high editing level being correlated with better patient survival times across cancer types. We demonstrated that in contrast to wild-type miRNA, edited miR-379-5p inhibited cell proliferation and promoted apoptosis in diverse tumor contexts in vitro, which was due to the ability of edited but not wild-type miR-379$5 p$ to target CD97. Importantly, through nanoliposomal delivery, edited miR-379-5p mimics significantly inhibited tumor growth and extended survival of mice. Our study indicates a role of RNA editing in diversifying miRNA function during cancer progression and highlights the translational potential of edited miRNAs as a new class of cancer therapeutics.
\end{abstract}

\section{Introduction}

Adenosine-to-inosine (A-to-I) RNA editing is a widespread posttranscriptional mechanism in humans that is catalyzed by adenosine deaminase acting on RNA (ADAR) enzymes and introduces specific nucleotide changes at the RNA level without altering DNA sequence $(1,2)$. Computational analyses of RNA-Seq data have detected a large number of RNA editing sites in the human transcriptome (3-5). More recently, several studies have systematically characterized the RNA-editing genomic landscape across cancer lineages using sequencing data from The Cancer Genome Atlas (TCGA) (6-8), revealing many dysregulated A-to-I RNA editing events in tumors. Some of the RNA editing events occur in coding regions, modify encoded amino acids, and directly affect cancer cell growth, suggesting "driver mutation"-like behaviors $(7,9,10)$.

The vast majority of A-to-I RNA editing events occur in noncoding regions, such as UTRs, introns, long non-coding RNAs, and microRNAs (miRNAs) (11-13). RNA editing in miRNAs is of particular interest since these approximately 22-nucleotide small RNAs play critical regulatory roles in multiple cellular pathways and patho-

Authorship note: $\mathrm{XX}, \mathrm{YW}, \mathrm{KM}$, and $\mathrm{ZZ}$ contributed equally to this work. Conflict of interest: $\mathrm{HL}$ is a shareholder in and scientific advisor to Precision Scientific Ltd. and Eagle Nebula Inc. AKS serves on advisory boards to Kiyatec and Merck, receives research funding from $\mathrm{M}$-Trap, and is a shareholder in Biopath. Copyright: ( 2019, American Society for Clinical Investigation. Submitted: July 6, 2018; Accepted: May 29, 2019; Published: November 4, 2019 Reference information: J Clin Invest. 2019;129(12):5343-5356. https://doi.org/10.1172/JCl123396. logical processes $(14,15)$. If editing occurs within the seed sequence of a miRNA, since a single nucleotide change can alter the base pairing properties of miRNA, this could alter recognition of target genes by deleting original targets or acquiring new targets. Intriguingly, several miRNA editing events appear to be critical negative regulators of cancer development (16). For example, edited miR-376a inhibits glioma cell migration and invasion via targeting of $A M F R$, combined with an inability to inhibit the original target RAP2A (17). Edited miR-455 inhibits melanoma tumor growth and metastasis by upregulating the tumor suppressor CPEB1 (18). Edited miR-589$3 p$ inhibits glioblastoma cell proliferation, migration, and invasion by retargeting the oncogene ADAM12 from the tumor suppressor $P C D H 9$ (19). MiR-378a editing can prevent melanoma progression through the regulation of PARVA expression (20). However, studies of these miRNA editing events have been limited to single diseases (i.e., glioma or melanoma), and the potential therapeutic value of edited miRNAs has not been explored in a broader context.

We recently performed a large-scale analysis of miRNA editing hotspots across 20 cancer types using TCGA miRNA-Seq data (21). An RNA editing event in the seed region of miR-379-5p showed intriguing patterns across cancer types, leading us to characterize its functional consequences. Herein, we characterize the effects of edited miR-379-5p, elucidate its molecular mechanism in diverse tumor contexts, and, importantly, assess its therapeutic potential in vivo through a clinically relevant, weight-dependent dosage of edited miR-379-5p using a recent nanoliposomal delivery platform. Our study highlights the potential prognostic and therapeutic utility of edited miR-379-5p. 


\section{Results}

A-to-I editing in miR-379-5p shows clinically relevant patterns across cancer types. We first validated the A-to-I RNA editing in miR-379$5 \mathrm{p}$ (Figure 1A) by performing ADAR-perturbed miRNA-Seq experiments. In cancer cell lines, we observed a dramatic increase in the editing level of miR-379-5p only upon ADAR2 overexpression (Supplemental Figure 1; supplemental material available online with this article; https://doi.org/10.1172/JCI123396DS1), indicating that this editing event is catalyzed specifically by the ADAR2 enzyme.

To assess the potential biomedical significance of miR-379$5 p$ editing, we next examined its editing patterns in both tumor and normal samples using TCGA miRNA-Seq data (Supplemental Table 1). The edited miR-379-5p showed relatively high expression levels across different normal tissues (e.g., 40 reads per million in breast tissue). To provide a more biologically relevant context, we assessed the expression of edited miR-379-5p relative to all 2588 human wild-type (WT) miRNAs (Figure 1B). In 9 of 13 tissue types examined, the expression amount of edited miR-379$5 \mathrm{p}$ ranked among the top 200 miRNAs. Since miRNAs with the highest expression level are believed to maintain key cellular functions and have significant impact on their corresponding target genes $(22,23)$, this result suggests that the highly expressed edited miR-379-5p is likely to play a functional role in gene regulation in normal tissues. We subsequently examined miR-379-5p editing in tumor samples and found that compared with matched normal samples, this editing site showed a consistent hypo-editing pattern in all 7 cancer types, with statistical significance (paired $t$ test, $q<$ 0.05 ; Figure $1 \mathrm{C}$ ). Thus, editing of miR-379-5p is frequently inhibited in tumor cells. To assess the potential relevance of decreased RNA editing in tumors, we assessed correlations of miR-379-5p editing level with patient survival times. Interestingly, higher miR379-5p editing levels were significantly correlated with better patient survival in several cancer types, including head and neck squamous cell carcinoma, kidney renal clear cell carcinoma, lung adenocarcinoma, and thyroid cancer (Figure 1D). Moreover, even taking tumor stage and patient age into account as potential confounding factors, correlations of miR-379-5p editing with clinical outcomes remained significant (Figure 1E), suggesting potential prognostic value independent from standard clinical variables. These patterns, observed over diverse TCGA patient cohorts, strongly suggest that miR-379-5p editing acts as a tumor suppressor event and that the decreased RNA editing level in tumor cells may be related to tumor initiation or progression.

Edited miR-379-5p strongly inhibits cell proliferation through induced apoptosis. To investigate the functional impact of miR379-5p editing, we used RNA mimics to examine its effects on cell proliferation in several cell lines representing diverse tumor contexts (MDA-MB-231 for breast cancer, OVCAR-8 for ovarian cancer, 786-O for renal cancer, and A549 for lung cancer). These cell lines were selected based on TCGA clinical relevance, feasibility for in vivo experiments, and the baseline level of miR-379-5p. WT miR-379-5p showed no significant effects on cell proliferation in comparison with the control mimics (Figure 2A), which shows good agreement with a previous study (24). In contrast, expression of edited miR-379-5p in each cell line dramatically decreased cell proliferation by IncuCyte proliferation assays in 2-dimensional (2D) cell cultures (ANOVA followed by Tukey's test, $P<0.05$;
Figure 2A). Next, we examined the effects of edited miR-379-5p on cell proliferation using colony formation assays and found that edited miR-379-5p markedly inhibited colony formation in each cell line (ANOVA followed by Tukey's test, $P<0$.05; Figure 2, B and $C$ ). We further performed cell viability assays in 3D cultured cancer cells and, as observed in 2D experiments, found that edited miR-379-5p significantly decreased cancer cell survival and proliferation in terms of relative viability, perimeter, and area (ANOVA followed by Tukey's test, $P<0.05$; Figure 2, D and E). In addition, cells transfected with edited miR-379-5p exhibited decreased migration and invasion (Supplemental Figure 2). These results suggest that edited miR-379-5p is a strong tumor suppressor of cancer cell survival and proliferation.

To further identify the pathways altered by edited miR-379-5p, we investigated its effects on apoptosis and the cell cycle. In each of the cancer cell lines studied, annexin V (AV) binding revealed an increase of early apoptosis, $\mathrm{AV}^{+}$propidium iodide-negative $\left(\mathrm{PI}^{-}\right)$ staining, and late apoptosis, $\mathrm{AV}^{+} \mathrm{PI}^{+}$staining, in cells transfected with edited miR-379-5p but not in cells transfected with negative control or WT miR-379-5p (ANOVA followed by Tukey's test, $P<$ 0.05; Figure 3, A and B). In terms of the cell cycle, we observed that edited miR-379-5p reduced the $G_{0} / G_{1}$ population in most cell lines, concomitant with a marked increase in the $G_{2} / M$ subpopulation in MDA-MB-231 and OVCAR-8, which was consistent with cell death in these cell lines (Supplemental Figure 3). Taken together, these results suggest that edited miR-379-5p inhibits cell proliferation by promoting apoptosis and may affect the cell cycle in some tumor contexts.

A-to-I editing directs miR-379-5p to a new set of target genes. A-to-I editing in miR-379-5p can potentially change its target genes since the RNA editing occurs in the seed region. To systematically identify key targets of edited miR-379-5p, we used RNA-Seq to identify genes that were significantly downregulated across 5 cell lines (paired $t$ test, $q<1 \times 10^{-5}$ ) upon transfection of WT or edited miR-379-5p mimics (relative to the control mimics). To assess the efficiency of miRNA overexpression, we performed gene set enrichment analysis to test whether the potential miRNA target genes were enriched in downregulated genes. Indeed, we found that miRDB-predicted WT miR-379-5p targets were significantly enriched in the downregulated genes in WT miRNA versus the negative control and, importantly, predicted edited miR-379$5 p$ targets that were significantly enriched in downregulated genes in edited mimics versus the negative control (Figure 4A and Supplemental Figure 4). We further identified high-confidence target candidates by integrating differential expression and binding motif information (WT miR-379-5p targets should have the binding motif GUCUACC in their 3'-UTRs; edited miR-379-5p targets should have GUCCACC in their 3'-UTRs), and found that only 2 genes overlapped between these 2 predicted target gene sets. This global analysis suggests that the single-nucleotide editing of miR-379-5p indeed redirects the miRNA to inhibit a new set of target genes by altering the seed sequence complementarity. We next focused on individual targets of WT and edited miRNAs for detailed characterization.

The focal adhesion kinase PTK2 is a WT miR-379-5p target (24), with a single binding site for the WT miRNA seed (Figure 4A). We observed substantial inhibition of PTK2 at both mRNA 

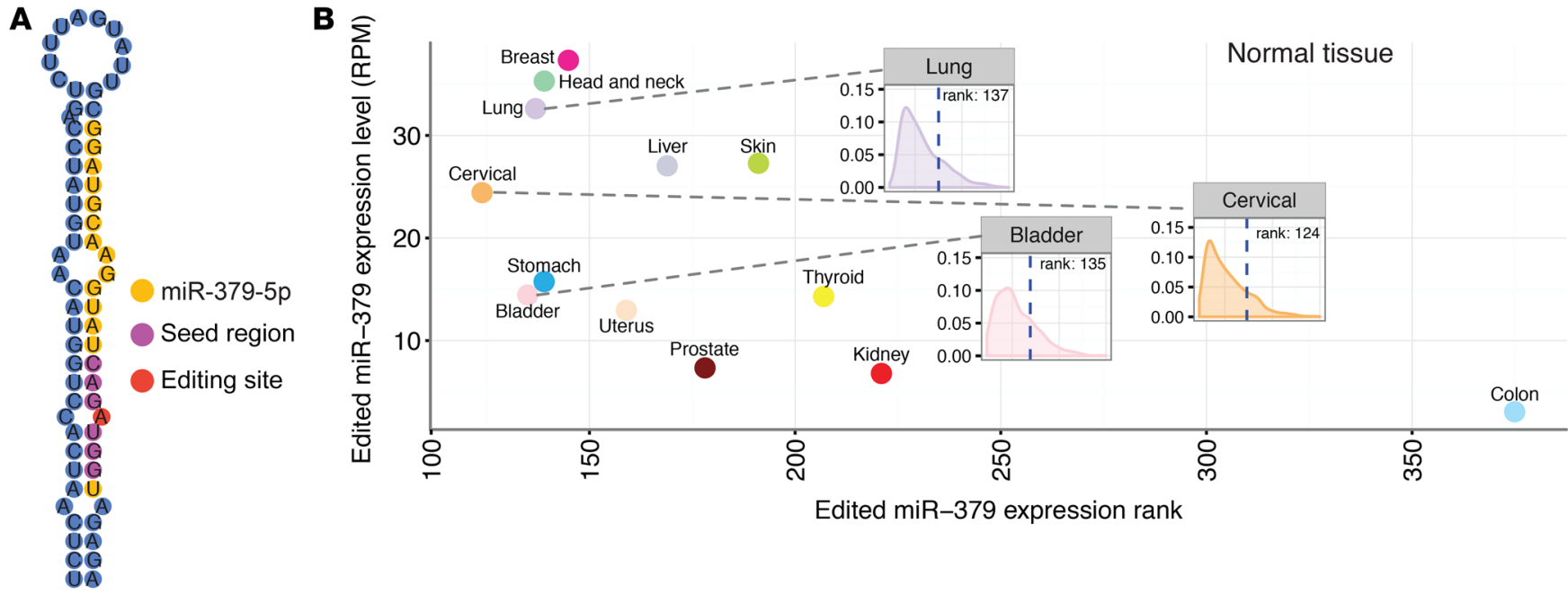

Edited miR-379 expression rank
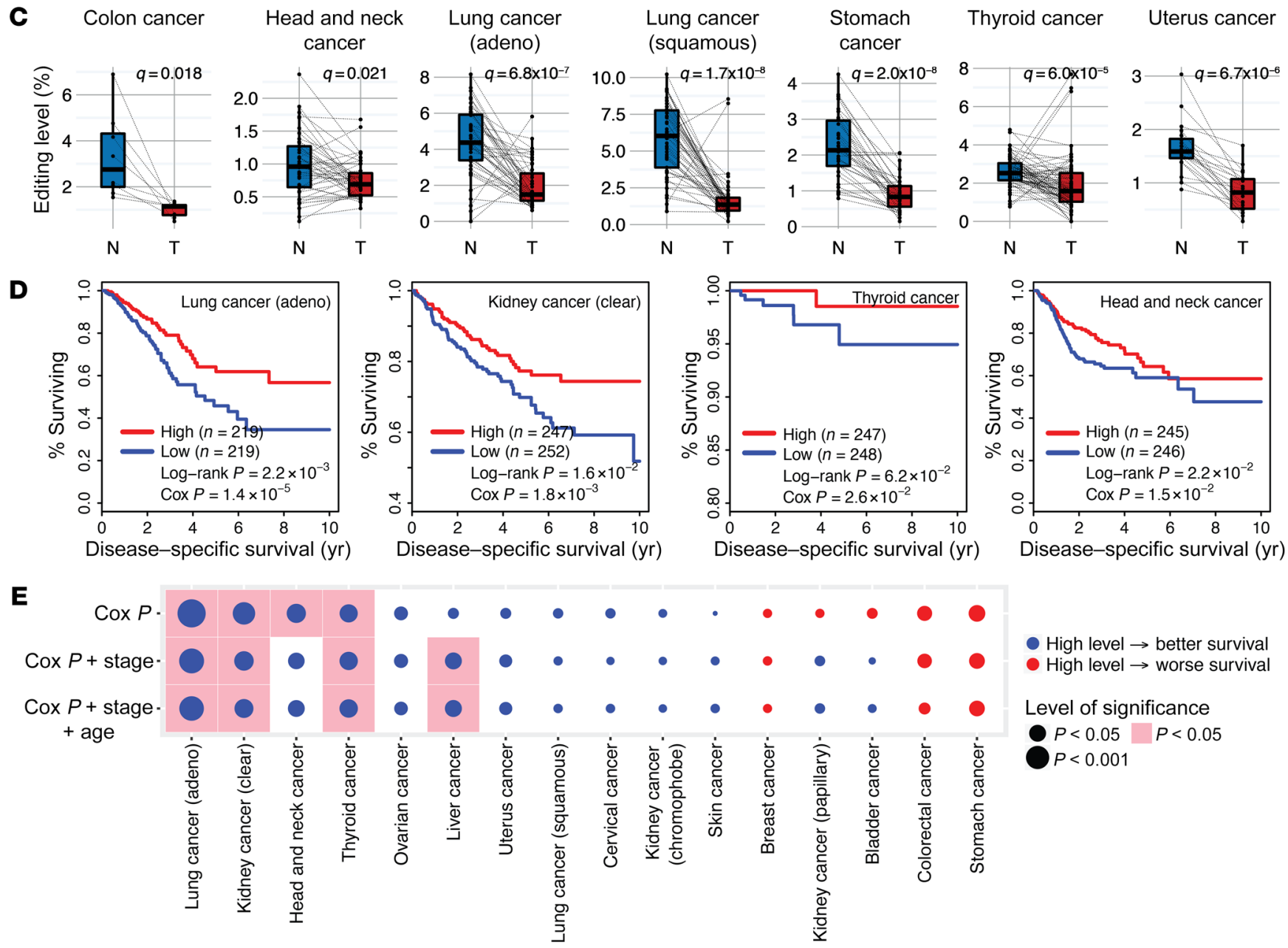

Figure 1. Clinically relevant patterns of miR-379-5p RNA editing in TCGA patient samples. (A) Schematic of RNA editing in miR-379-5p, showing the miRNA stem-loop (blue), mature miRNA (orange), seed region (purple), and editing site (red). (B) Expression amount of edited miR-379-5p in the context of WT miRNA expression in various normal tissues. Rank of edited miR-379-5p upper-quantile expression value relative to the median expression of all WT human miRNAs in different cancer types ( $x$ axis). Raw expression amount (reads per million mapped reads to miRNA [RPM]) of edited miR-379-5p ( $y$ axis). Magnified panels show the background distribution of WT miRNAs in representative cancer types. (C) Editing-level comparison between tumor samples and matched normal samples in the cancer types. Raw $P$ values based on paired Wilcoxon test; statistically significant differences ( $q<0.05$ ) are reported. The middle line in the box is the median, the bottom and top of the box are the first and third quartiles, and the whiskers extend to 1.5 times the interquartile range of the lower and the upper quartiles, respectively. (D) Significant survival correlations of miR-379-5p editing level with patient disease-specific survival times in different cancer types $(P<0.05)$; $P$ values based on Cox proportional hazards models. The groups are separated by median values. (E) Summary of Cox proportional hazard ratio model of miR-379-5p editing level with patient disease-specific survival. Dot size represents level of statistical significance; color indicates correlation direction. Pink blocks highlight $P<0.05$. Stage and age as confounding factors are included in the model for comparison. 

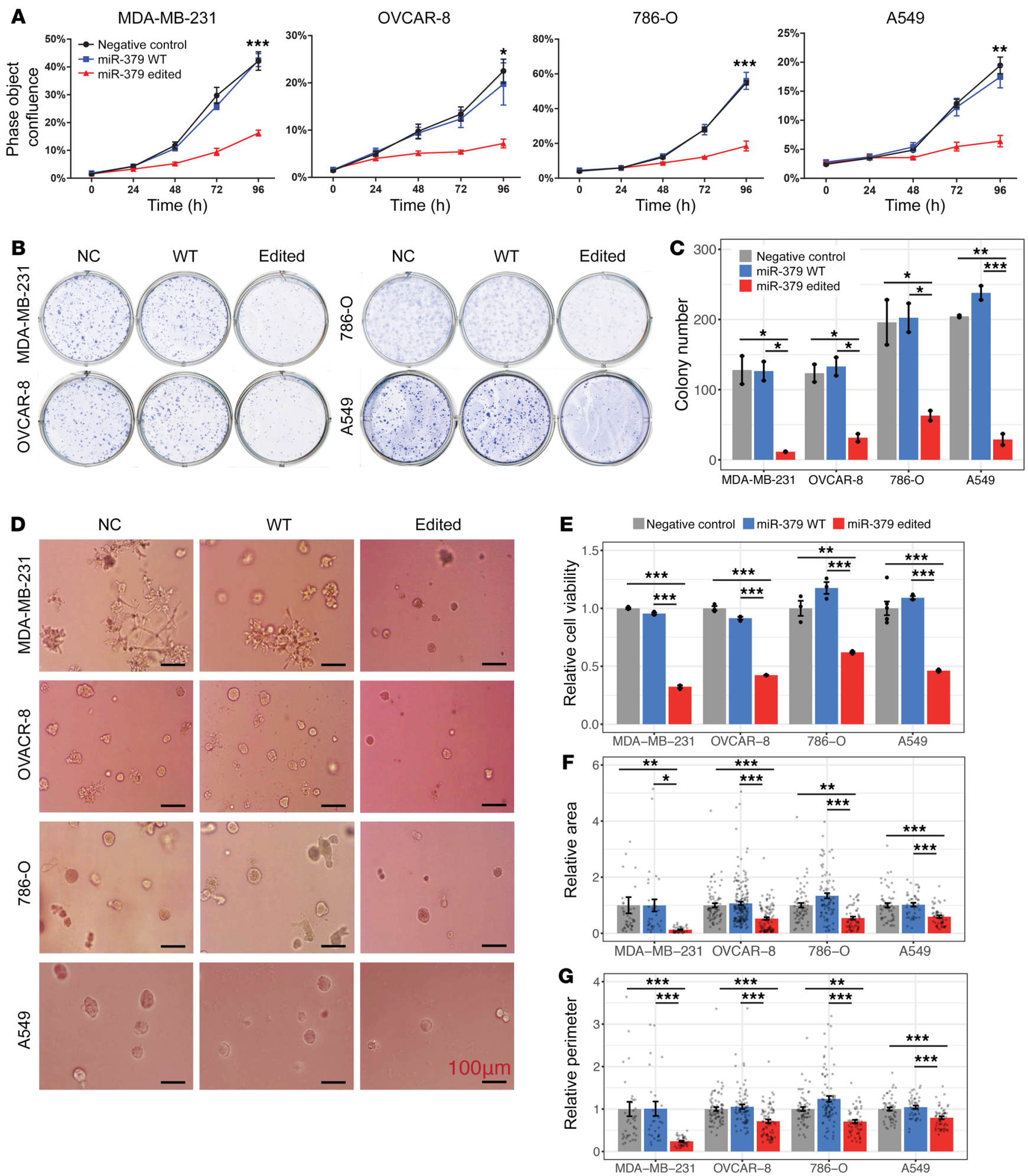

Figure 2. Effects of RNA editing in miR-379-5p on cell proliferation and clone formation. (A) Effects of edited miR-379-5p mimics on cell viability in $2 \mathrm{D}$ cultured MDA-MB-231, OVCAR-8, 786-0, and A549 cells by IncuCyte proliferation assays. (B and C) Clone formation assays transfected with negative control, WT, and edited miR-379-5p mimics (B), and summary of the clone number (C). (D-G) Representative images of cell viability assays transfected with negative control, WT, and miR-379-5p mimics in 3D cultured cell lines (D); and summary of relative cell viability (E), area (1 outlier, 11.25 in MDA-MB-231 negative control, not shown) (F), and perimeter (2 outliers, 5.88 in MDA-MB-231 negative control and 4.09 in MDA-MB-231 WT, not shown) (C). All data are based on 3 independent experiments. Error bars denote \pm SEM; ANOVA followed by Tukey's test, ${ }^{*} P<0.05 ;{ }^{* *} P<0.01 ;{ }^{* *} P<0.001$. 
and protein levels for cells treated with WT miR-379-5p but not with edited miR-379-5p (Figure 4, B and C). The pattern was consistent across cancer cell lines, indicating that PTK2 is a target gene of WT but not edited miR-379-5p.

To identify target genes of edited miR-379-5p, we prioritized candidate genes from the RNA-Seq experiments using the following criteria: (a) rank in the top 15 most significantly decreased genes and $\log _{2}$ fold change greater than 1.5 in both edited miR-379$5 p$ versus negative control and edited miR-379-5p versus WT miR379-5p; (b) has at least 1 binding site for edited miR-379-5p but no binding site for WT miR-379-5p; and (c) has a detectable baseline expression level in the cell lines surveyed. This yielded 2 candidate targets of edited miR-379-5p: adhesion G protein-coupled receptor E5 (CD97, also known as ADGRE5) and G protein subunit $\alpha \mathrm{I} 2$ (GNAI2). CD97 has a single binding site in its 3 '-UTR (Figure 4A). Indeed, edited miR-379-5p dramatically reduced the expression of CD97 at both mRNA and protein levels in all 4 cancer cell lines (ANOVA followed by Tukey's test, $P<0.01$; Figure 4, D and E). In contrast, WT miR-379-5p had little effect on CD97 expression (Figure 4, D and E). We further verified direct binding of edited miR-379-5p with CD97 3'-UTR using a luciferase reporter assay. We cotransfected mimics with a luciferase reporter containing the predicted target site, and observed that edited miR-379-5p mimics inhibited luciferase expression (ANOVA followed by Tukey's test, $P<0.01$; Figure 4F), whereas cotransfection with negative control or WT miR-379-5p mimics did not show such effects (Figure 4F). Furthermore, there was a strong negative correlation between the edited miR-379-5p level and CD97 mRNA expression across different groups of TCGA tumor samples $(R s=-0.83, P=0.006)$ (Supplemental Figure 5). Collectively, these results indicate that A-to-I editing in miR-379-5p creates a neomorphic miRNA molecule that loses the ability to repress its original targets such as PTK2 but acquires the ability to target a new set of genes such as CD97.

Edited miR-379-5p confers its phenotypic effects through regulation of CD97. CD97 is a marker for minimal residual disease in acute lymphoblastic leukemia (25), and also a leukemic stem cell marker in acute myeloid leukemia (26). This gene has been reported to be induced or upregulated in a variety of human cancers (27-30); the overexpression of CD97 was found to enhance local tumor growth in immune-deficient mice (30). In addition, shRNA-mediated knockdown of CD97 has been shown to increase caspase-mediated apoptosis $(31,32)$. Clinically, high expression of CD97 correlates with worse patient survival times across cancer types (Supplemental Figure 6). Therefore, we focused on CD97 for further investigation.

To examine whether edited miR-379-5p confers its phenotypic effects through silencing of CD97, we performed a set of in vitro experiments. We first decreased $C D 97$ expression using 3 CD97-specific siRNAs and confirmed their knockdown effects by Western blot analyses (Supplemental Figure 7). We then performed functional assays using 2 of the verified siRNAs (\#1 and \#2). We found that the biological effects of CD97 knockdown copied the phenotype effects of edited miR-379-5p and resulted in similar enhanced apoptosis and obvious reduction in proliferation of the 4 cancer cell lines (ANOVA followed by Tukey's test, $P$ $<0.05$; Figure 5, A-C, and Supplemental Figure 8), suggesting that CD97 functions as a suppressor of cancer cell apoptosis. In con- trast, there was no effect on proliferation and apoptosis in these cancer cells through transfection of the other candidate target GNAI2 siRNAs (data not shown). Thus, CD97 could explain the effect of edited miR-379-5p on cell apoptosis.

To further demonstrate that the enhanced apoptosis phenotype was indeed due to the direct interaction of edited miR379-5p with CD97, rather than indirect consequences caused by secondary modulation of $C D 97$, we tested whether $C D 97$ expression could rescue the effects of edited miR-379-5p on cellular proliferation. We used 2 overexpression constructs for $C D 97$ : one with the full-length CD97 cDNA containing both CD97 ORF and 3 '-UTR (named CD97 CDNA) and the other containing the CD97 ORF only, without the 3'-UTR (named CD97 ORF). The constructs were cotransfected with negative control, WT miR379-5p, or edited miR-379-5p into MDA-MB-231 and OVCAR-8 cells. We confirmed that the $C D 97$ protein was downregulated by edited miR-379-5p only when CD97 cDNA (i.e., containing the 3'-UTR) was expressed, whereas negligible downregulation of CD97 occurred when CD97 ORF (not containing the 3'-UTR) was expressed (Figure 5, D and E). Importantly, the effects of edited miR-379-5p on proliferation were not markedly compromised by the presence of $C D 97 \mathrm{cDNA}$, while concomitant overexpression of the CD97 ORF (which is not downregulated by the edited miRNA) abrogated the inhibition of cell proliferation induced by edited miR-379-5p (ANOVA followed by Tukey's test, $P<0.05$; Figure 5, $\mathrm{F}$ and $\mathrm{G}$ ). Our findings indicate that $C D 97$ is an immediate downstream effector specific to edited miR-379-5p and is likely to be a major target gene through which the proliferation-inhibiting and apoptosis-prompting effects of edited miR-379-5p are mediated.

Edited miR-379-5p is a potential cancer therapeutic in vivo. To explore the potential therapeutic utility of edited miR-379-5p in human cancers, we performed mouse experiments to assess its inhibitive effects on tumor growth. We first established an in vivo breast cancer model using luciferase-labeled MDA-MB-231 cells. We then examined the therapeutic efficacy of edited miR-379-5p using 1,2-dioleoyl-sn-glycero-3-phosphatidylcholine-mediated (DOPC-mediated) small RNA delivery technology. DOPC is a recently developed neutral liposome carrier for the delivery of oligonucleotides (33). Administration of small RNAs using DOPC delivery is currently under investigation in clinical trials (34). To mimic the amount of the DOPC complex that can be delivered in clinical trials, mice were treated with edited miR-379-5p incorporated in DOPC twice a week for 4 weeks (Figure 6A). We found that treatment with edited miR-379-5p mimics significantly decreased tumor volumes compared with negative control and WT miR-3795p groups in MDA-MB-231 ( $P=0.02$ and $P=0.04$, respectively) (Figure 6, B and C, and Supplemental Figure 9). Further, mice treated with edited miR-379-5p showed significantly improved survival times compared with negative control mice and mice treated with WT miR-379-5p (3-group log-rank $P=0.02$, edited vs. others $\log$-rank $P=0.01$, respectively; Figure 6D). We observed similar inhibitory effects of edited miR-379-5p on tumor growth in another xenograft model based on A549 cells (Figure 6E).

We also studied the effects of edited miR-379-5p on MDAMB-231 xenografts. Consistent with the effects of edited miR$379-5 p$ on the growth of cancer cells in vitro, we found that edited miR-379-5p decreased the expression of CD97 and increased 
A

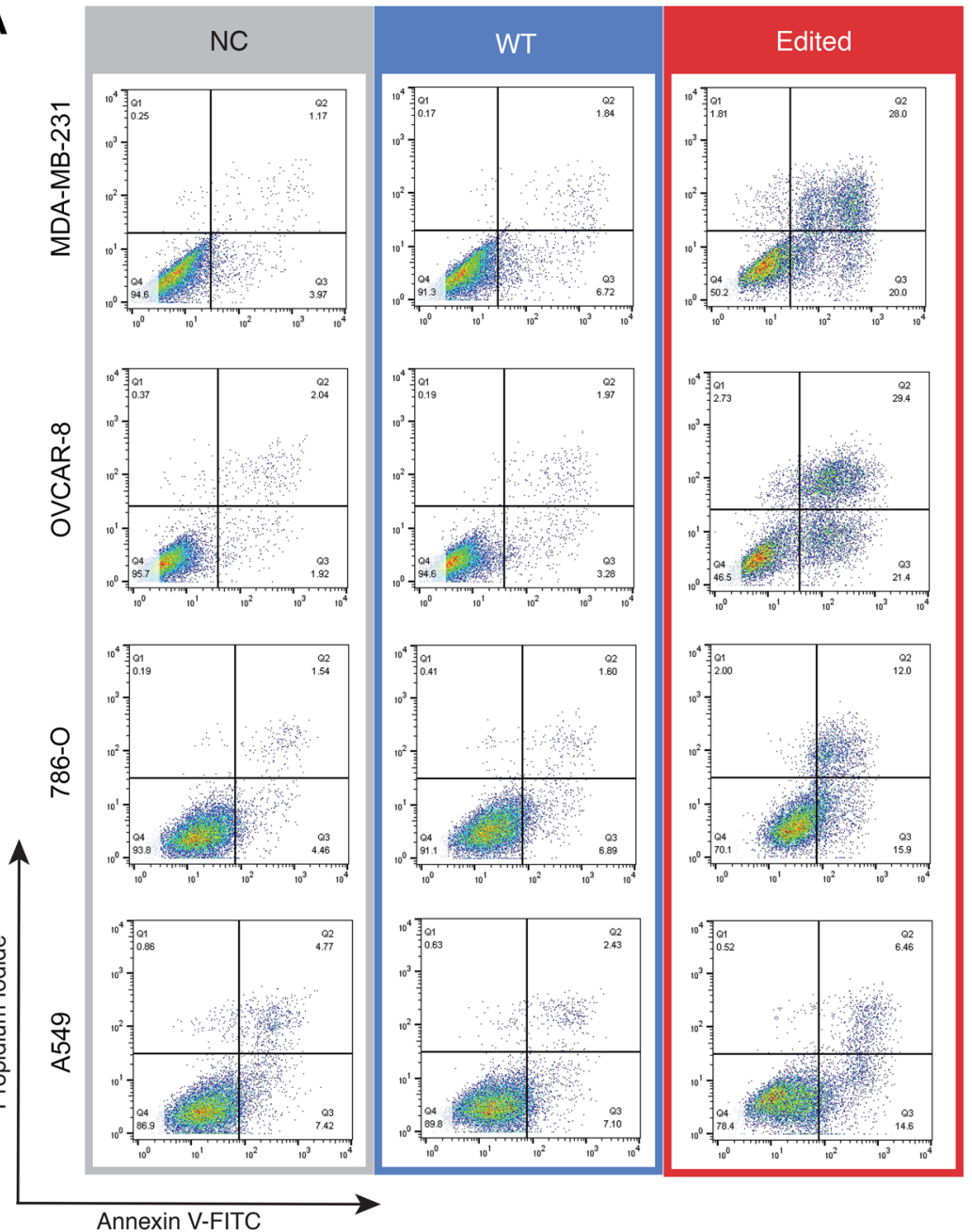

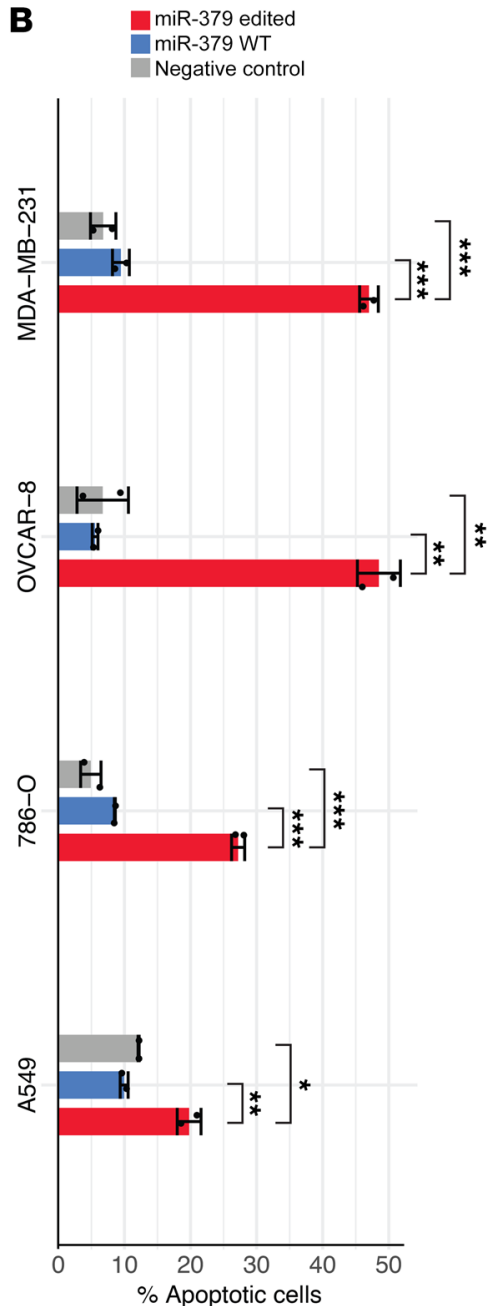

Figure 3. Effects of edited miR-379-5p on cell apoptosis. (A) Apoptosis of miRNA mimic-transfected cells by AV-FITC/PI staining. (B) Measurement of total death of early-apoptotic $\mathrm{AV}^{+} \mathrm{Pl}^{-}$and late-apoptotic $\mathrm{AV}^{+} \mathrm{Pl}^{+}$cells transfected with negative control, WT, and miR-379-5p mimics. Results are based on 3 independent experiments. Error bars denote \pm SD; ANOVA followed by Tukey's test, ${ }^{*} P<0.05 ;{ }^{* *} P<0.01 ;{ }^{* *} P<0.001$.

the amount of the apoptotic cell marker cleaved caspase-3 (Supplemental Figure 10, A and B). In addition, edited miR-379-5p reduced the level of the Ki-67 proliferation marker in tumor tissues (Supplemental Figure 10, B-D).

Taken together, the data provide a model for the role of edited miR-379-5p in cancer: Following processing of pre-miR-379 and subsequent modification by the ADAR2 enzyme, edited miR-3795 p binds to the $C D 97$ 3'-UTR based on sequence complementarity and inhibits its expression at both mRNA and protein levels. Subsequently, decreased amounts of CD97 lead to activation of a caspase- 3 cascade that induces cell apoptosis and inhibits cell proliferation. Thus, a single nucleotide modification converts miR-379-5p into a suppressor of tumor growth. Our results in vivo further suggest a therapeutic potential of edited miR-379-5p for cancer treatment (Figure 7).

\section{Discussion}

A single nucleotide change induced by editing of miRNA can markedly alter function. Here, we focused on an A-to-I editing event in miR-379-5p and characterized its functional conse- quences. Unlike previously reported edited miRNAs, edited miR379-5p shows clinically relevant patterns across multiple cancer types, and our functional assays confirmed its phenotypic effects in diverse tumor contexts. This intriguing pattern provides strong rationale for further exploring diagnostic, prognostic, and possibly therapeutic strategies.

CD97 is a prototypic member of the adhesion class of G protein-coupled receptors, which play essential roles in developmental processes and tumorigenesis $(22,35)$. CD97 is induced, upregulated, and/or biochemically modified in various malignancies compared with the corresponding normal tissues $(30,36)$. CD97 expression is inversely related with the overall survival times of patients with glioblastoma and gallbladder carcinoma $(37,38)$. The binding partners of CD97 include CD55, chondroitin sulfate $\mathrm{B}$ $(39,40)$, integrin $\alpha_{5} \beta_{1}$, and CD90 $(41,42)$. In thyroid and prostate cancer cells, CD97 amplifies LPAR1 signaling to promote tumor progression in a Rho-dependent fashion $(30,43)$. CD97 has also been shown to inhibit programmed tumor cell death (31). All these previous findings are well aligned with the mechanistic model we propose in which the functional impact of edited miR-379-5p is at 

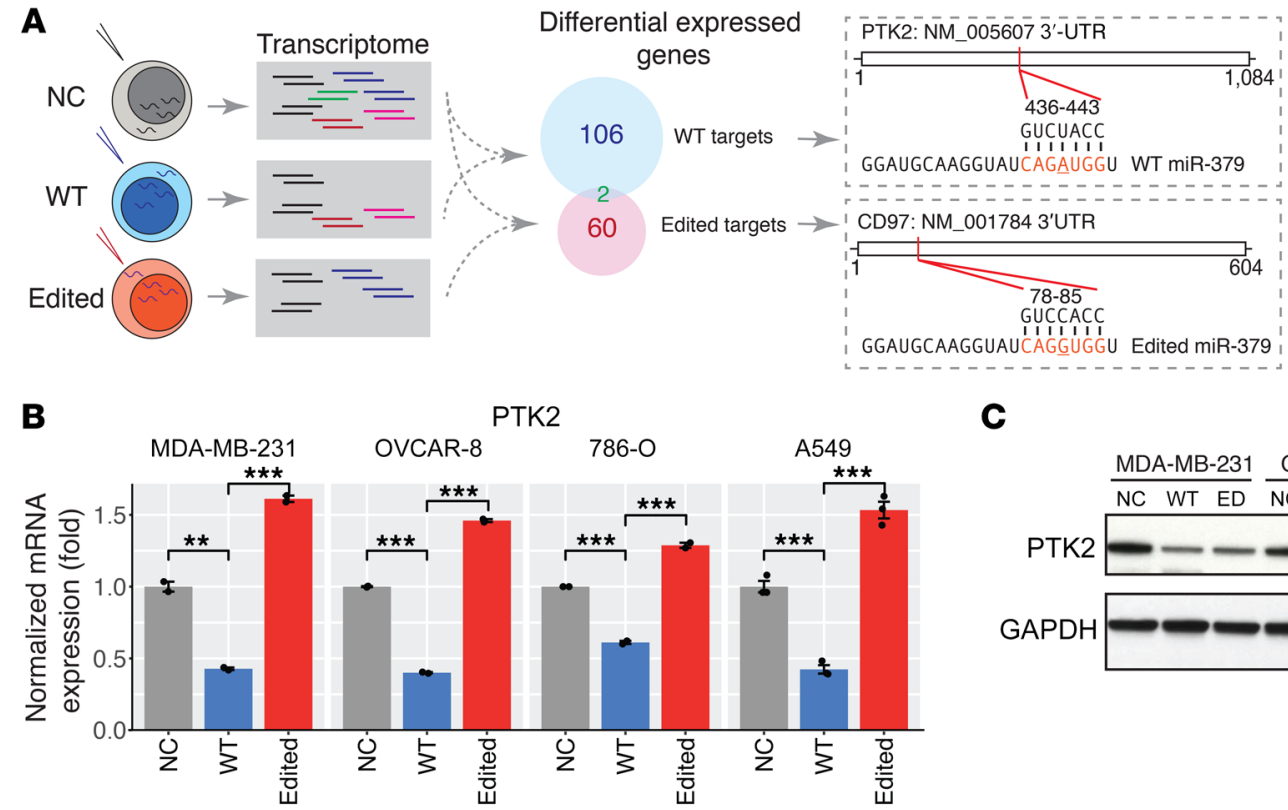

C
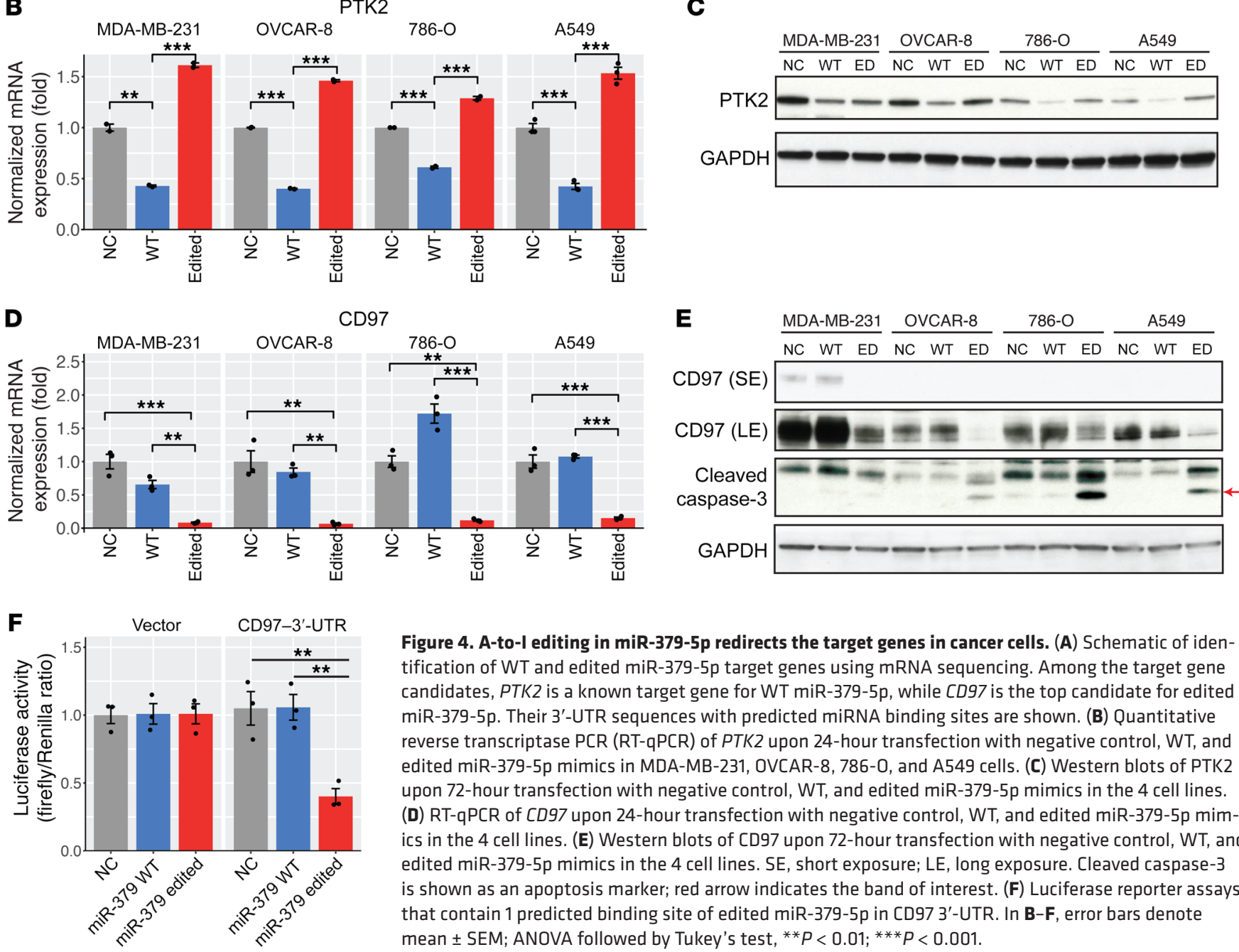

Figure 4. A-to-I editing in miR-379-5p redirects the target genes in cancer cells. (A) Schematic of identification of WT and edited miR-379-5p target genes using mRNA sequencing. Among the target gene candidates, PTK2 is a known target gene for WT miR-379-5p, while CD97 is the top candidate for edited miR-379-5p. Their 3'-UTR sequences with predicted miRNA binding sites are shown. (B) Quantitative reverse transcriptase PCR (RT-qPCR) of PTK2 upon 24-hour transfection with negative control, WT, and edited miR-379-5p mimics in MDA-MB-231, OVCAR-8, 786-0, and A549 cells. (C) Western blots of PTK2 upon 72-hour transfection with negative control, WT, and edited miR-379-5p mimics in the 4 cell lines. (D) RT-qPCR of CD97 upon 24-hour transfection with negative control, WT, and edited miR-379-5p mimics in the 4 cell lines. (E) Western blots of CD97 upon 72-hour transfection with negative control, WT, and edited miR-379-5p mimics in the 4 cell lines. SE, short exposure; LE, long exposure. Cleaved caspase-3 is shown as an apoptosis marker; red arrow indicates the band of interest. (F) Luciferase reporter assays that contain 1 predicted binding site of edited miR-379-5p in CD97 $3^{\prime}-U T R$. In B-F, error bars denote mean \pm SEM; ANOVA followed by Tukey's test, ${ }^{* *} P<0.01$; ${ }^{* *} P<0.001$.

least partially due to its acquired ability to target $C D 97$, thereby inhibiting proliferation and increasing apoptosis.

One concern may be that our functional results were based on exogenous overexpression studies in which the edited miRNA level may be higher than the endogenous level. But one major goal of this study was to investigate the translational potential of edited miRNAs as therapeutics. From this perspective, it is less important whether the endogenous level of edited miR-379-5p is function$\mathrm{al}$; and further efforts are needed to assess the contribution of the endogenously reduced edited miR-370-5p to tumor development. In order to assess antitumor activity, we used a DOPC nanoliposomal delivery platform for systemic delivery of edited miR-379-5p for in vivo experiments and demonstrated significant therapeutic activity on primary tumors in multiple mouse models. In vivo delivery of oligonucleotides in experimental models has shown promise for use in humans. Liposomal delivery of drugs is an established and safe approach, and their use for siRNA or miRNA delivery makes this therapeutic modality clinically attractive. DOPC loaded with antisense oligonucleotides against Grb2 are currently in phase I clinical trials in leukemia, and another clinical trial based on siRNA-EphA2-DOPC for patients with ovarian cancer is ongoing at MD Anderson Cancer Center (44). Further efforts should be made to investigate whether edited miR-379-5p delivered by DOPC is effective for a specific patient subpopulation such as those with high CD97 expression or whether it can be combined with other anticancer therapies to enhance therapeutic efficacy. In a broad 


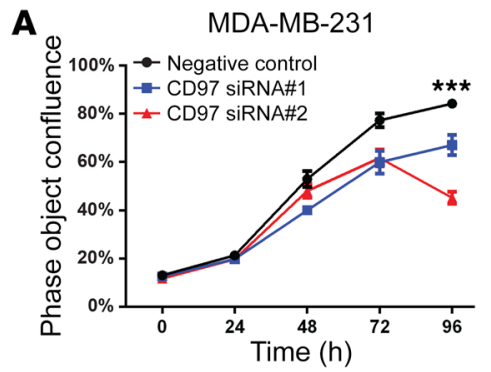

OVCAR-8

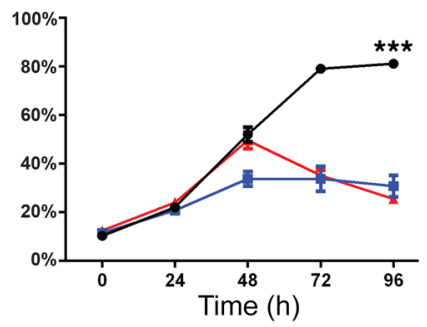

786-O

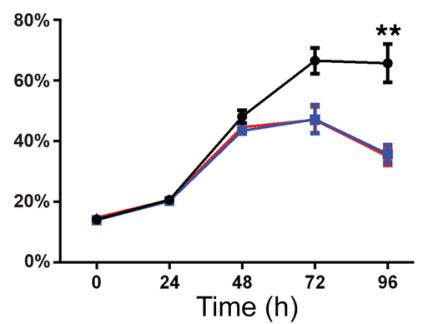

A549

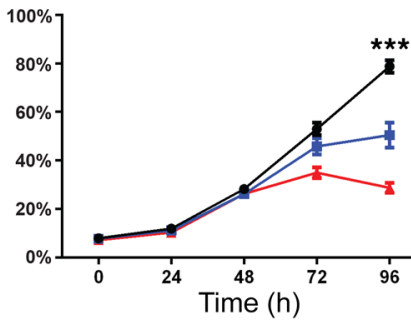

B

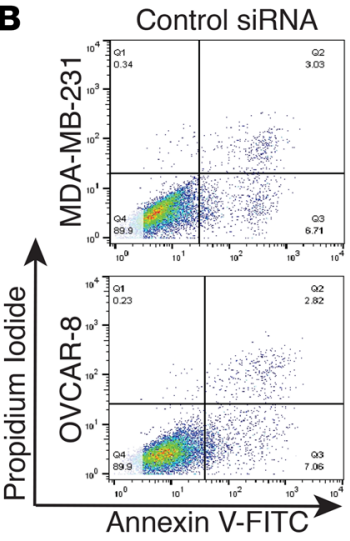

D
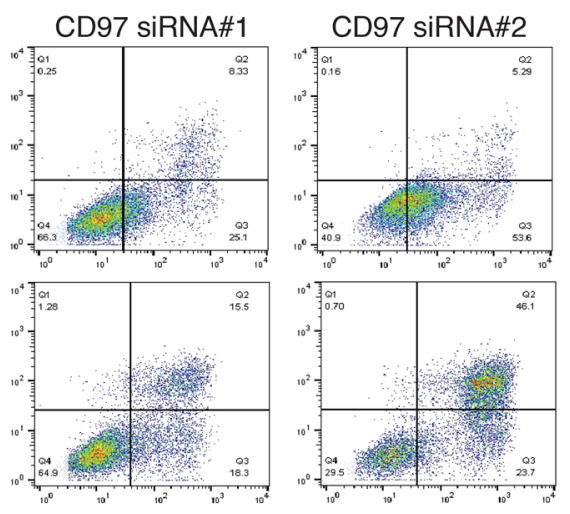

C

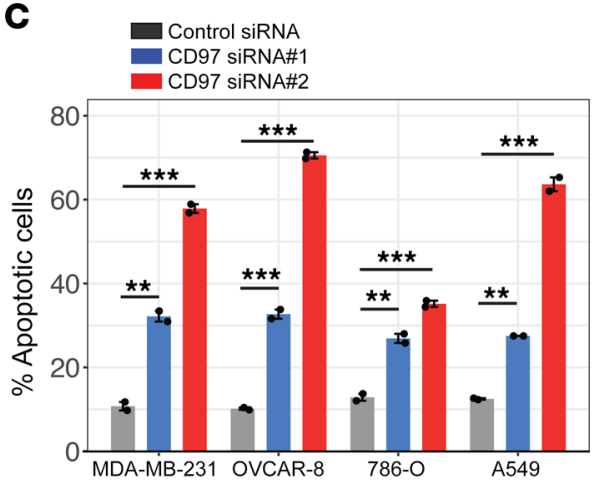

$+-\quad+\quad-\quad-$

$\begin{array}{llllll}+ & - & - & + & - & - \\ - & + & - & - & + & - \\ - & - & + & - & - & + \\ - & - & - & - & - & - \\ + & + & + & - & - & - \\ - & - & - & + & + & +\end{array}$

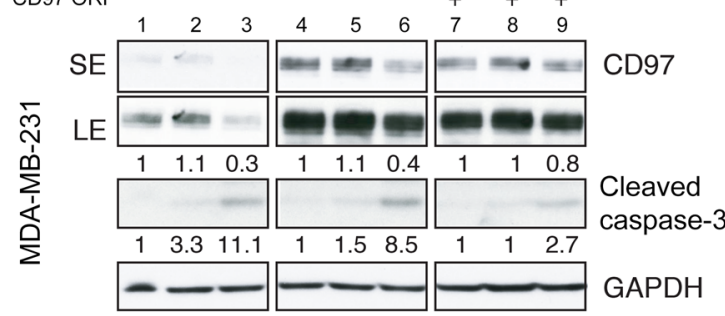

$\mathbf{F}$

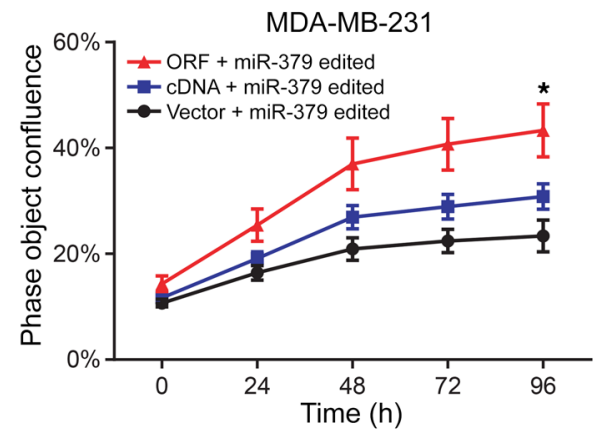

E

$\begin{array}{rccccccccc}\text { Control } & + & - & - & + & - & - & + & - & - \\ \text { miR-379 WT } & - & + & - & - & + & - & - & + & - \\ \text { miR-379 edited } & - & - & + & - & - & + & - & - & + \\ \text { pCMV-vector } & + & + & + & - & - & - & - & - & - \\ \text { CD97 cDNA } & - & - & - & + & + & + & - & - & - \\ \text { CD97 ORF } & - & - & - & - & - & - & + & + & + \\ & 1 & 2 & 3 & 4 & 5 & 6 & 7 & 8 & 9\end{array}$

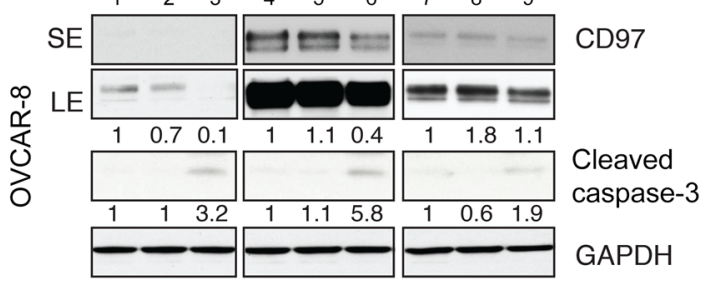

G

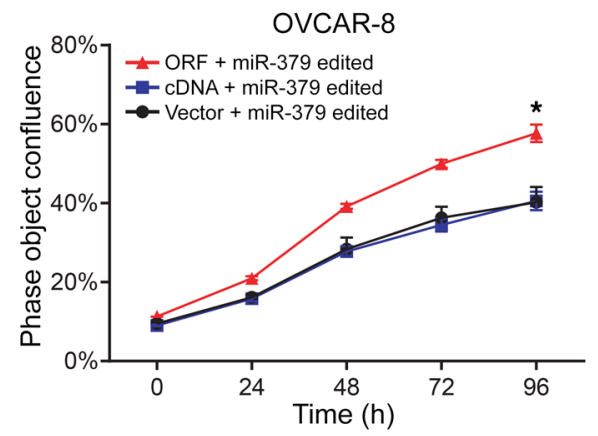

Figure 5. Edited miR-379-5p confers its phenotypic effects through CD97. (A) Effects of CD97 siRNAs (\#1 and \#2) on cell proliferation in MDAMB-231, OVCAR-8, 786-0, and A549 cells. Two-sided $t$ test was used to assess the difference. (B) Representative images of cell apoptosis after transfection of 2 CD97 siRNAs (\#1 and \#2) by AV-FITC/PI staining. (C) Effects of 2 CD97 siRNAs (\#1 and \#2) on cell apoptosis by AV-FITC/PI staining. Error bars denote \pm SEM; ANOVA followed by Tukey's test, ${ }^{*} P<0.05$; ${ }^{* *} P<0.01 ;{ }^{* *} P<0.001$. (D and E) Western blots of CD97 and cleaved caspase-3 in MDA-MB-231 (D) and OVCAR-8 (E) cells, cotransfected with CD97 expression vectors and WT, edited miR-379-5p, or control miRNAs. GAPDH was used as loading control. Densitometric analysis was done by ImageJ software, and values were normalized to control for each construct. (F and $\mathbf{G})$ IncuCyte proliferation assay of MDA-MB-231 (F) and OVCAR-8 (G) cells cotransfected with expression vectors containing empty vector control (pCMV-vector), a full-length CD97 cDNA (CD97 cDNA), or CD97 without 3'-UTR (CD97 ORF) and edited miR-379-5p. Error bars indicate SD; ANOVA followed by Tukey's test, ${ }^{*} P<0.05 ;{ }^{* *} P<0.01 ;{ }^{* *} P<0.001$. 
A

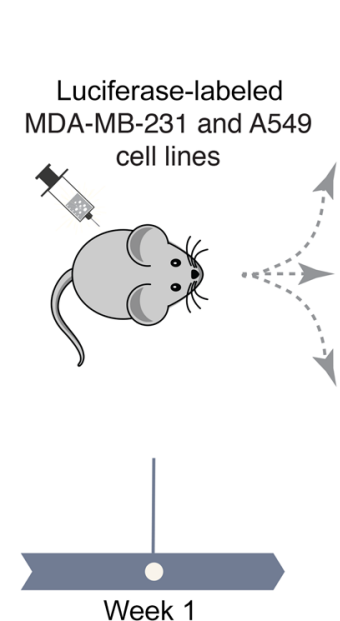

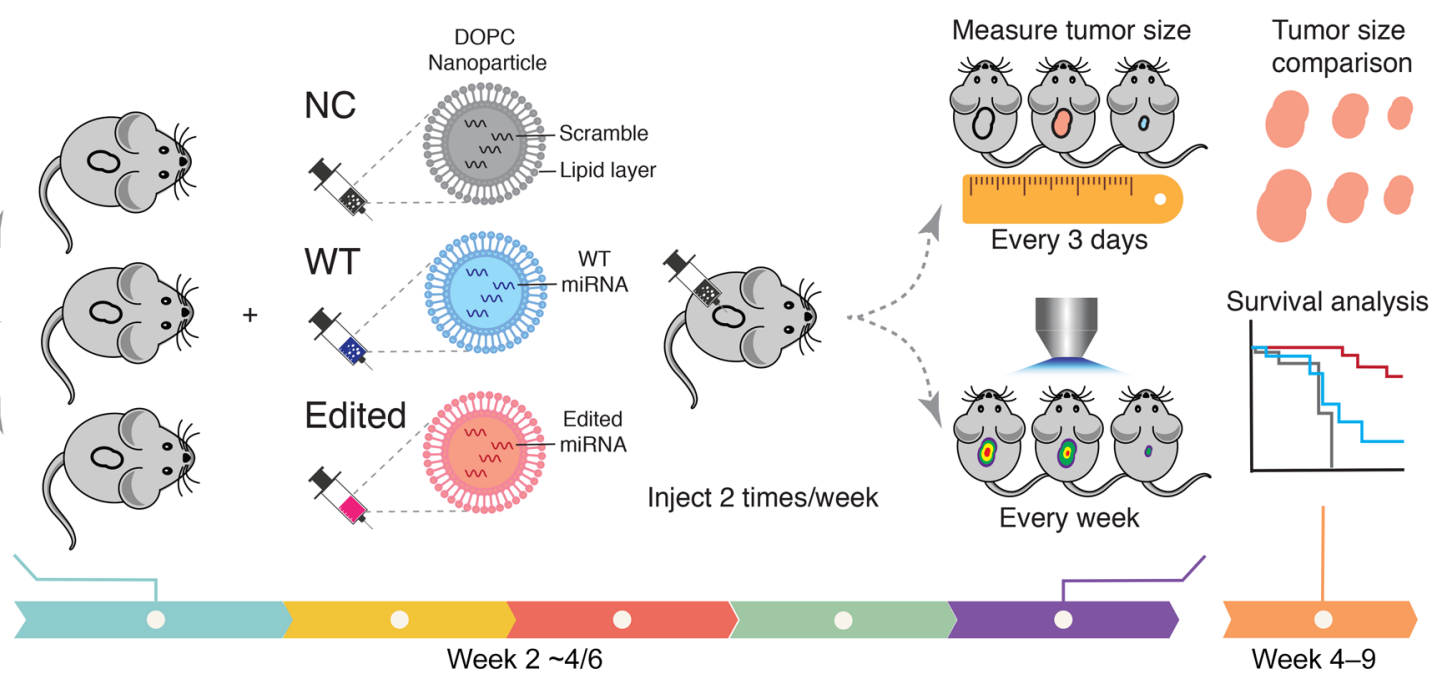
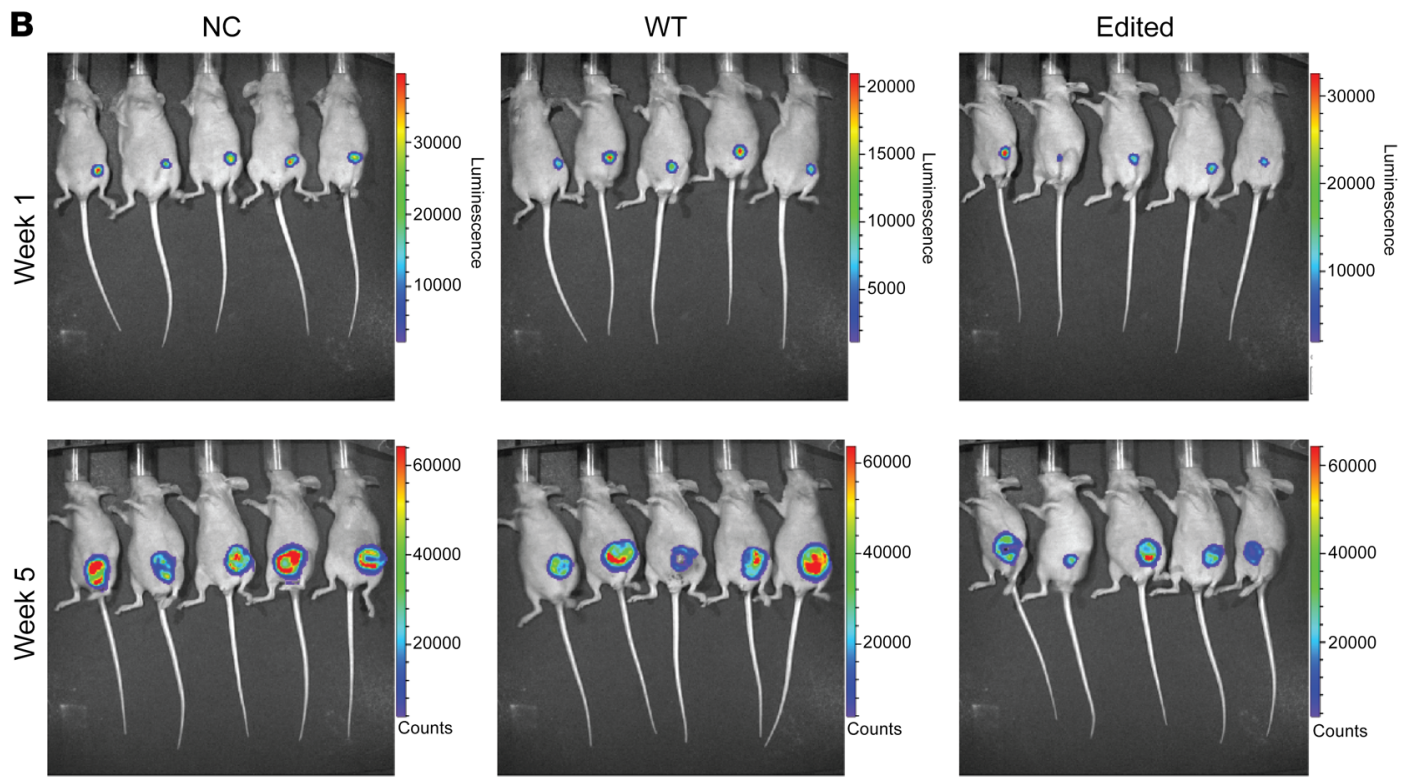

C

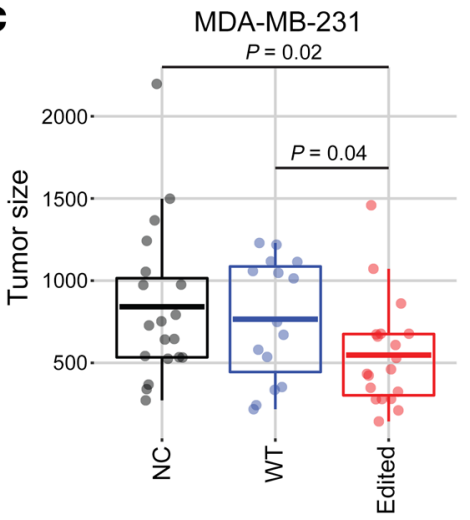

D

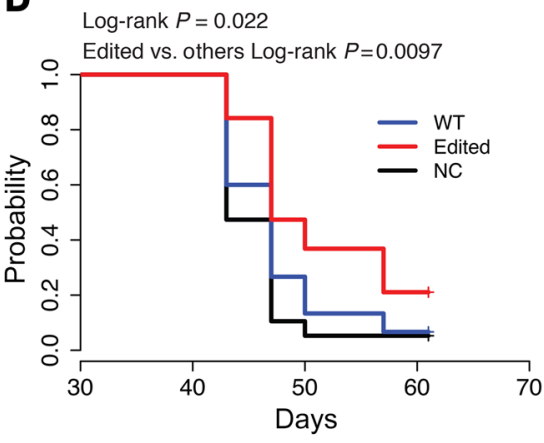

E

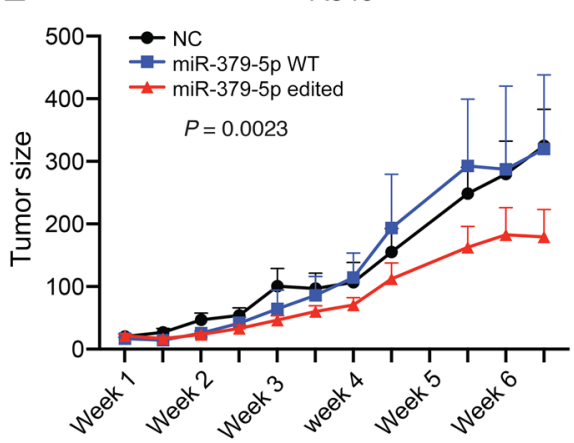

Figure 6. Therapeutic effects of edited miR-379-5p in vivo. (A) Flowchart of in vivo experiments. The MDA-MB-231 cell line was used in the xenograft model. MiRNA mimics incorporated into DOPC nanoliposomes were injected as treatment twice a week. Tumor sizes were measured every 3 days using a caliper, and a luciferase picture was taken once a week. (B) In Vivo Imaging Systems images of the mice at the first and fifth weeks. (C) Box plots of tumor sizes measured by caliper of MDA-MB-231 in vivo model at week $4.5 ; t$ test was used to assess the statistical difference. The middle line in the box is the mean, the bottom and top of the box are the first and third quartiles, and the whiskers extend to 1.5 times the interquartile range of the lower and the upper quartiles, respectively. (D) Kaplan-Meier survival curves for different mouse groups of MDA-MB-231 in vivo model; $P$ values based on log-rank tests. (E) Tumor sizes (mean with SEM) measured by caliper of A549 in vivo model. A 2-way ANOVA test was used to evaluate the impact of time and different treatments. Each measurement was considered independent, and treatment $P$ value was reported. 


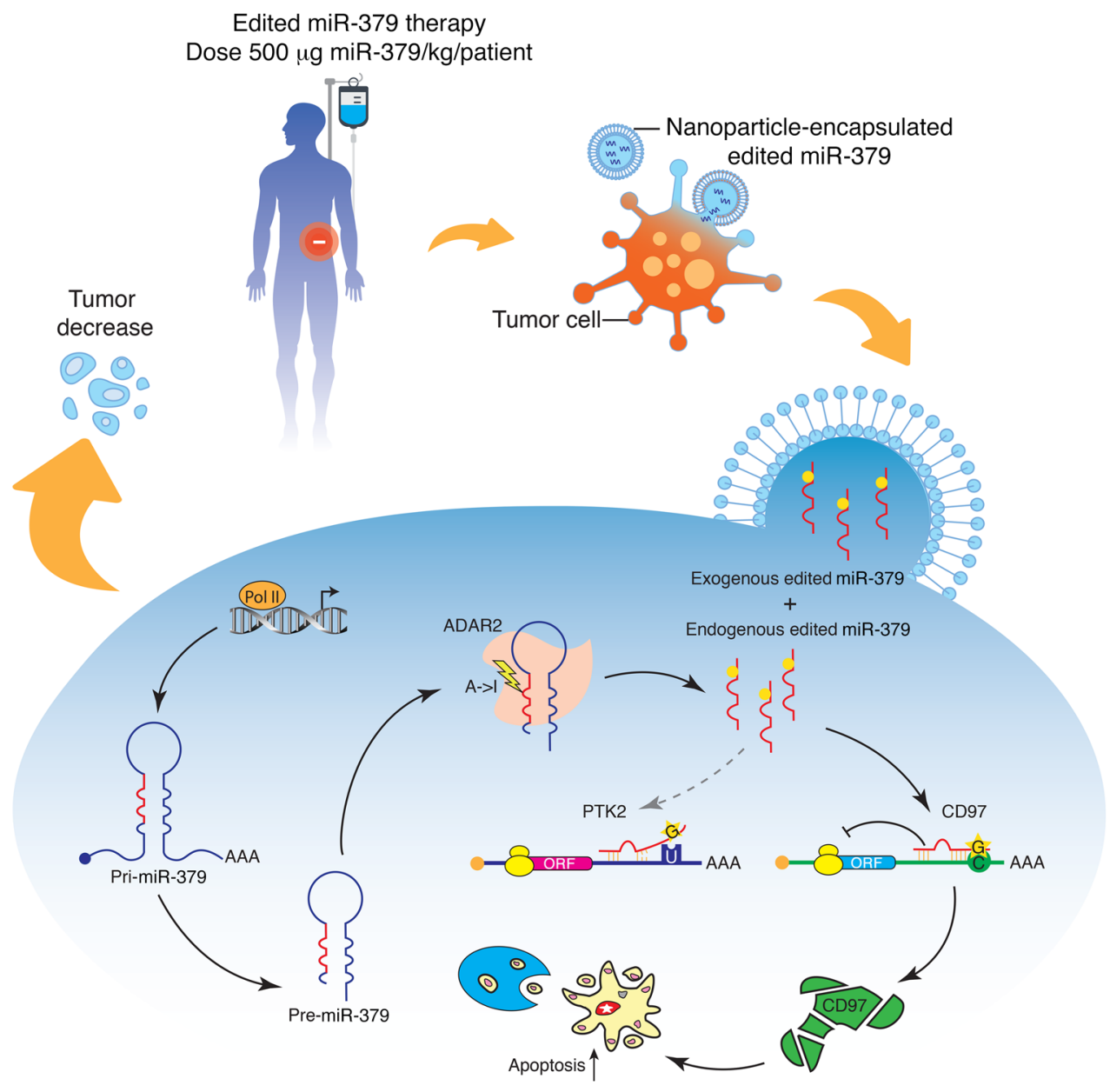

Figure 7. Proposed mechanistic model and potential clinical utility of edited miR-379-5p. Nanoparticle-encapsulated edited miR-379-5p can be administered to patients with a dose of $500 \mu \mathrm{g} / \mathrm{kg}$. Endogenous and exogenous edited miR-379-5p (catalyzed by ADAR2) inhibits tumor growth by targeting the apoptosis regulator CD97 (a promoter of tumor cell apoptosis), which leads to tumor shrinkage. POL II, RNA polymerase II. sense, edited miRNA such as miR-379-5p could represent a novel cancer therapeutic strategy, especially for traditionally undruggable targets. Its effectiveness across cancer types makes this potential even more striking. In the future, with the advent of RNA-based CRISPR technology (45), such modified miRNAs may provide an exciting class of therapeutics warranting investigation (46).

\section{Methods}

Bioinformatics analysis of miR-379-5p editing in cancer. We obtained TCGA miRNA-sequencing BAM files of 634 normal samples and 7961 tumor samples from National Cancer Institute (NCI) Genomic Data Commons (GDC). Using our previously published pipeline (21), we calculated the miRNA editing level (defined as the percentage of edited reads among all the reads mapped to the position of interest) and expression amount (defined as the edited reads per million mapped reads $[\mathrm{RPM}]$ ) per sample. To assess the relative expression of edited miR-379-5p for a given cancer type, we first calculated the median expression values (RPM) of all expressed WT mature miRNAs $(n=$ 2588) across the tumor samples, then measured the upper-quartile expression value of edited miR-379-5p for samples with detectable editing signals, and ranked it against the background expression distribution of WT miRNAs. For cancer types with at least 10 pairs of matched tumor-normal samples, we compared the editing level of miR-379-5p using paired Wilcoxon test and assessed the statistical significance with the false discovery rate. To assess clinical signifi- cance, we obtained TCGA patient clinical data, including patient age, pathological stage, and disease-free survival time from NCI GDC. We performed 3 analyses to test whether the editing level of miR-379-5p correlated with the patient's disease-free survival time: (a) the univariate Cox proportional hazards model (47); (b) the multivariate Cox proportional hazards model taking patient age as an independent factor; and (c) the multivariate Cox proportional hazards model taking both patient age and pathological stage as independent factors.

Cell culture and transfection. MDA-MB-231, OVCAR-8, 786-O, Hs 578T, HeyA8, PC-3, and HEK293 cells were obtained from MD Anderson Characterized Cell Line Core Facility. The A549 cell line was purchased from American Type Culture Collection. All the cell lines were confirmed by short tandem repeat analysis, and mycoplasma testing was found to be negative. HEK293 cells were cultured in DMEM that contained $4500 \mathrm{mg} / \mathrm{L}$ glucose supplemented with $10 \%$ FBS; and the others were cultured in RPMI 1640 medium supplemented with 10\% FBS.

The mutant ADAR1 and ADAR2 ORFs (ADAR1-E912A and ADAR2E396A) were made by site-directed mutagenesis and confirmed by Sanger sequencing as previously described (7). These mutant ORFs contain an E-to-A amino acid change that abolishes ADAR editase activity (48). Viruses were produced by transfection of HEK293 cells with the green fluorescent protein (GFP) control vectors, pHAGE-V5-puromycin expression vectors (carrying ADAR1-WT or ADAR1-E912A; ADAR2WT or ADAR2-E396A), and the lentiviral packaging plasmids psPAX2 and pMD2.G. Hs 578T and 786-O cells were transduced by the virus fol- 
lowed by selection with puromycin (Hs 578T, $1 \mu \mathrm{g} / \mathrm{mL}$; and 786-O, $3 \mu \mathrm{g}$ / $\mathrm{mL}$ ), and after 7 days of antibiotic selection, expression of the constructs was verified by Western blots.

MiRNA mimics were purchased from Sigma-Aldrich (USA): MISSION miRNA Negative Control 1 (HMC0002) and MISSION microRNA Mimic hsa-miR-379-5p (HMIO547). The sense sequence of hsa-miR-379-5p edited mimics is [AmC6F] CCUACGUUCCAUAGUCCAUCCAdTdT, and the antisense sequence is UGGUGGACUAUGGAACGUAGGdTdT. Cells were transfected with $25-50 \mathrm{nM}$ of the indicated miRNA mimics using Lipofectamine RNAiMAX reagent (13778150, Thermo Fisher Scientific).

Three siRNAs against CD97 (CD97 siRNA \#1: SASI_Hs01 00209256; CD97 siRNA \#2: SASI_Hs01_00209257; and CD97 siRNA \#3: SASI_Hs01_00209258) were purchased from Sigma-Aldrich. Each siRNA targeted a distinct region of target mRNA. MISSION siRNA universal negative control \#1 from Sigma-Aldrich was used as control siRNA.

Full-length CD97 expression constructs including the 3'-UTR (pCMV6-XL4-CD97 cDNA) were purchased from Origene (cata$\log$ SC109040). To express CD97 ORF only, expression constructs (pCMV6-entry-CD97 ORF) were also purchased from Origene (catalog RC202001). A control vector was generated from pCMV6-entry-CD97 ORF by SgfI/MluI double digestion and inserted the sequence that could not target any known vertebrate gene (GAAATGTACTGCGCGTGGAGACGTTTTGGCCACTGACTGACGTCTCCACGCAGTACATTT). Cotransfection of miRNA mimics (25-50 nM per well of 6-well plates) and expression constructs ( $2 \mu \mathrm{g}$ DNA) was conducted with Lipofectamine RNAiMAX and Lipofectamine 3000 reagent (Invitrogen).

$R N A$ isolation and quantitative real-time reverse transcriptase PCR. To examine the effects on the potential miRNA target genes, total RNAs were isolated using RNeasy Plus Mini Kit (Qiagen). RNAs were transcribed into cDNAs using the High-Capacity cDNA Reverse Transcription Kit (Life Technologies). Reactions were done in duplicate using TaqMan Fast Universal PCR Master Mix (2×), No AmpErase UNG (Life Technologies), or SYBR Select Master Mix (Life Technologies). The primers 5'-3' from Thermo Fisher Scientific were as follows: PTK2 primers (Hs01056457_m1), $\beta$-actin primers (Hs99999903_m1), CD97 primers (forward: GAAATTAAAGAAGGCGAGGG; reverse: GTAAACACATAGGTCAGCAC), and $\beta$-actin primers (forward: ATTGGCAATGAGCGGTTCCG; reverse: CGTGGATGCCACAGGACTCC). Quantitative reverse transcriptase PCR was performed by Applied Biosystems 7900HT Fast Real-Time PCR system (Applied Biosystems). The relative expression was calculated by the $2^{-\Delta \Delta \mathrm{Ct}}$ method. Expression levels were normalized to $\beta$-actin.

Immunoblotting. Whole-cell lysates for Western blotting were extracted with RIPA buffer (25 mM Tris- $\mathrm{HCl}$ pH 7.6, $150 \mathrm{mM} \mathrm{NaCl}, 1 \%$ NP-40, $1 \%$ sodium deoxycholate, $0.1 \%$ SDS, protease, and phosphatase inhibitor cocktail). Protein concentrations were determined using Pierce BCA protein assay kit (catalog 23225, Thermo Fisher Scientific) assays according to the manufacturer's instructions. Cell lysates (20 $\mu \mathrm{g})$ were loaded onto 4\%-12\% Criterion XT Bis-Tris Protein Gel (Bio-Rad) and transferred to a PVDF membrane, and protein expression was depicted with an ECL Western blot detection kit (Amersham Biosciences). The following antibodies were used: CD97 [EPR4427] (1:1000; ab108368, Abcam), PTK2 (1:1000; CST-3285, Cell Signaling Technology), V5 (1:5000; R960-25, Life Technologies), caspase-3 (31A1067) (1:500; sc-56053, Santa Cruz Biotechnology), and GAPDH (1:3000; sc-25778, Santa Cruz Biotechnology).
IncuCyte proliferation assays. MDA-MB-231, OVCAR-8, 786-O, and A549 cell lines were seeded into 6-well plates, and transfected with miRNA mimics. After transfection for 24 hours, cells were plated in 96-well plates, and proliferation was monitored by analysis of the occupied area (\% confluence) of cell images over time. As cells proliferate, the confluence increases. Confluence is an excellent surrogate for proliferation, until cells are densely packed or when large changes in morphology occur. The graphs from the phase of cell confluence area were recorded at $0,24,48,72$, and 96 hours according to the IncuCyte (Essen BioScience) manufacturer's instructions.

Colony formation assay. Transfected cells were seeded into 6-well plates (1000 cells per well) and cultured for 1 week. At the end of the growth period, cells were fixed with ethanol and stained with Coomassie brilliant blue R-250. The cell colonies were photographed, and the number of colonies was counted for statistical analysis.

3D Matrigel assay. Eight-well glass chamber slides (catalog 354108, BD Falcon) were coated with growth factor-reduced Matrigel (catalog 354230, BD Biosciences). Coated chambers were incubated at $37^{\circ} \mathrm{C}$ while cells were trypsinized and counted. Cells suspended in $2 \%$ Matrigel solution were added to coated chamber slides. Cells were maintained 8 days in an incubator at $37^{\circ} \mathrm{C}, 5 \% \mathrm{CO}_{2}$ with fresh medium containing $2 \%$ Matrigel added every 2 days. We monitored the morphologies by taking images. For cell viability assays, $10 \mu \mathrm{L}$ PrestoBlue Cell Viability Reagent (catalog A13261, Thermo Fisher Scientific) was added directly to the well with $100 \mu \mathrm{L}$ cultured cells transferred from the chamber slides, and incubated at $37^{\circ} \mathrm{C}$ for 10 minutes or longer based on the total cell number. We recorded fluorescence using a microplate reader with excitation/emission (nm) 530/604. The analysis of the relative perimeter and area was processed by ImageJ software (NIH).

Migration and invasion assays. Cells were transfected with miRNA mimics as described above. Twenty-four hours after transfection, cells $\left(3.5 \times 10^{4}\right.$ to $\left.4.5 \times 10^{4}\right)$ were plated on uncoated (Corning BioCoat Control Insert; $8.0 \mu \mathrm{m}$; 24-well; 24/CS 354578, migration assay) or Matrigel-coated (Corning BioCoat Matrigel Invasion Chamber; 24-well; 24/CS 354483, invasion assay) Transwells in serum-free medium. Serum-supplemented medium was used as chemoattractant. Assays were allowed to proceed for 22-32 hours, at which time the invading/migrated cells on the lower side of the Transwells were fixed and stained using crystal violet solution (Sigma-Aldrich). Ten random low-power $(\times 100)$ fields were photographed for each condition, and cells were counted using ImageJ software.

Apoptosis assay. The cell lines were transfected with miRNA mimics, siRNA, or constructs for 72-96 hours at indicated concentrations and stained with AV-FITC and PI (K101-100, Biovision). Apoptosis was analyzed by flow cytometry using a Becton Dickinson Flow Cytometer FACScan according to the manufacturer's protocol. Both early-apoptotic $\left(\mathrm{AV}^{+}\right.$and $\left.\mathrm{PI}^{-}\right)$and late-apoptotic $\left(\mathrm{AV}^{+}\right.$and $\left.\mathrm{PI}^{-}\right)$cells were included in the analyses.

Cell cycle analysis. Cells were fixed in $75 \%$ ethanol at $-20^{\circ} \mathrm{C}$ overnight. The cells were then washed with cold PBS and treated with 0.5 mL of FxCycle PI/RNase Staining Solution (catalog F10797, Thermo Fisher Scientific). Cell cycle profiles were analyzed by flow cytometry using the Becton Dickinson Flow Cytometer FACScan.

Analysis of edited miR-379-5p target genes. MDA-MB-231, 786-O, HeyA8, OVCAR-8, and PC-3 cells transfected with WT and edited miR379-5p mimics were subjected to mRNA sequencing (the sequencing platform was HiSeq4000 and the paired-end reads were at $2 \times 76 \mathrm{bp}$ ) 
at the MD Anderson Sequencing and Microarray Core Facility. We mapped FASTQ raw reads and performed differential gene expression analysis using TopHat2 alignment with default parameters (49) and HTSeq-count with mode "union" (50), followed by EdgeR (51). We predicted the target gene set of WT and edited miR-379-5p using miRDB (http://www.mirdb.org), and assessed the efficiency of miRNA overexpression by evaluating whether the predicted targets were enriched in downregulated genes using gene set enrichment analysis (52). We identified the new targets of edited miR-379-5p by comparing the expression of the paired samples of edited miR-379-5p and control mimics. High-confidence predicted targets of edited miR-379-5p were selected.

We obtained TCGA CD97 mRNA expression data from NCI GDC PanCanAtlas (https://gdc.cancer.gov/about-data/publications/ pancanatlas). Samples with sufficient coverage of miR-379-5p (reads aligned $\geq 10$ ) were included in the analysis. We first classified all the samples into 10 bins, and then used the median value of each bin to calculate the correlation between CD97 expression and miR-379-5p editing level. The Spearman rank correlation test was used to assess the significance.

Luciferase reporter assay. The human CD97 3'-UTR fragment (367 bp) was PCR-amplified from the genomic DNA and subcloned into the pMIR-REPORT luciferase construct using the following cloning primers: forward, 5'-GATTGGGAGCTCCATATGAAGGCGCATGGTTCTGGAC-3'; reverse, 5'-CCGGCACGCGTCAAAGACATGAGAGGAAAAGTCCAGGATG-3'. HEK293 cells of $50 \%$ confluence in 24-well plates were transfected with $50 \mathrm{nM}$ miRNA mimics by Lipofectamine RNAiMAX reagent. After 6 hours, the cultured medium was changed, and the pMIR-REPORT firefly luciferase reporter gene construct (50 ng) and $5 \mathrm{ng}$ of the pRL-TK Renilla luciferase construct (for normalization) were cotransfected per well using Lipofectamine 3000 reagent (Invitrogen). Cell extracts were prepared 48 hours after transfection, and the luciferase activity was measured using the Dual-Luciferase Reporter Assay System (E1910, Promega).

Xenograft tumor studies. MiRNA mimics for in vivo delivery were encapsulated into DOPC. DOPC and miRNA mimics were mixed in the presence of excess tertiary butanol at a ratio of 1:10 (wt/wt) miRNA/ DOPC. Tween-20 was added to the mixture in a ratio of 1:19 Tween-20/ miRNA-DOPC. The mixture was vortexed, frozen in an acetone/dry ice bath, and lyophilized. Before in vivo administration, this preparation was hydrated with PBS at room temperature at a concentration of 500 $\mu \mathrm{g}$ miRNA per kilogram per mouse injection (each mouse received 200 $\mu \mathrm{L}$ of DOPC-miRNA-PBS solution by the intraperitoneal route).

Six-week-old female J:NU, also known as outbred athymic nude, mice (stock 007850) were purchased from The Jackson Laboratory and housed under pathogen-free conditions. Outbred nude mice are immunodeficient tumor transplant hosts and the standard in vivo model for drug efficacy testing oncology. All studies were approved and supervised by the Institutional Animal Care and Use Committee of MD Anderson Cancer Center. For the breast cancer xenograft model, MDA-MB-231 cells $\left(5 \times 10^{6}\right)$ expressing luciferase in $200 \mu \mathrm{L}$ growth medium were injected into the fourth mammary fat pad. For the lung cancer xenograft model, A549 cells $\left(3 \times 10^{6}\right)$ in $100 \mu \mathrm{L}$ growth medium were injected subcutaneously into the right flank. To study the effect of miRNAs incorporated in DOPCs on tumor development, mice bearing tumors were randomly assigned to the following treatment groups 8 days after cell injection (MDA-MB-231, $n=20$ mice per group; A549, $n=8-10$ mice per group): (a) negative control miRNA-DOPC, (b) WT
miR-379-5p-DOPC, (c) edited miR-379-5p-DOPC. A dose of $500 \mu \mathrm{g}$ miRNA $/ \mathrm{kg} /$ mouse was packaged within DOPC nanoliposomes and delivered intraperitoneally at twice-weekly intervals as previously described $(33,53,54)$. This concentration was determined by the Center for RNA Interference and Noncoding RNAs at MD Anderson Cancer Center, which ensures efficient delivery and knockdown of target genes. The body weight of the 6-week-old female J:NU mouse was 19.84 \pm 1.01 $\mathrm{g}$, and for therapeutic experiments, a dose of $10 \mu \mathrm{g}$ miRNA per mouse injection was used. Twice-weekly treatments via intraperitoneal injections commenced 8 days after cell inoculation and continued until the mice were euthanized. Tumor size was measured every 3 days using a caliper, and tumor volume was calculated using the standard formula: $0.5 \times L \times W^{2}$, in which $L$ is the longest diameter and $W$ is the shortest diameter. For the luciferase-expressing MDA-MB-231 xenografts, weekly luciferase imaging was performed using the Xenogen IVIS 200 (PerkinElmer) system within 15 minutes following injection of $150 \mathrm{mg} /$ $\mathrm{kg}$ VivoGlo Luciferin (Promega) per mouse. Living Image 2.5 software was used to determine the regions of interest, and the average photon radiance $\left(\mathrm{p} / \mathrm{s} / \mathrm{cm}^{2} / \mathrm{sr}\right)$ was measured for each mouse. The endpoint for tumor monitoring was set at 4-6 weeks, based on tumor volume or the end of treatment duration. The person who provided animal care and measured tumor growth was blinded to the group allocation during all animal experiments and outcome assessment.

For survival analysis, mice were euthanized with carbon dioxide when they met the institutional euthanasia criteria for tumor size and overall health condition. Kaplan-Meier analysis was performed, and survival curves were generated using Prism 6 software (GraphPad).

After the mice were euthanized, tumors were harvested. The freshly dissected tumor tissues were frozen for lysate preparation and fixed in $4 \%$ buffered formalin overnight, washed with PBS, transferred to $70 \%$ ethanol, embedded in paraffin, sectioned, and stained with H\&E.

Immunohistochemistry. Samples were deparaffinized and rehydrated. Antigen was retrieved using $0.01 \mathrm{M}$ sodium citrate buffer ( $\mathrm{pH}$ 6.0) at a sub-boiling temperature for 10 minutes after boiling in a microwave oven. To block endogenous peroxidase activity, the sections were incubated with $3 \%$ hydrogen peroxide for 10 minutes. After 15 minutes of blocking in Background Sniper (BS966, Biocare Medical), the samples were incubated with antibody against Ki-67 (CRM325, Biocare Medical) or cleaved caspase-3 (CP229, Biocare Medical) at 1 hour at room temperature. The sections were incubated with a secondary antibody goat anti-rabbit IgG-HRP (ab6721, Abcam), and then incubated and visualized with a DAB chromogen kit (DB801, Biocare Medical) according to the manufacturer's protocol. Counterstaining color was carried out using hematoxylin. All immunostained slides were scanned on the Image J software for quantification by digital image analysis.

Data access. The RNA sequencing data from this study were submitted to the NCBI Sequence Read Archive (https://www.ncbi.nlm. nih.gov/sra) under accession number SRP075557.

Statistics. Editing-level comparisons between matched tumor and normal samples were performed with paired Wilcoxon test. Adjusted $P$ value $(q)$ was reported. Patient survival analysis was performed with (univariate or multivariate) Cox proportional hazards model and logrank test. Cox model $P$ value and log-rank test $P$ value were reported. All in vitro experiment results were compared using ANOVA followed by Tukey's test for multiple test corrections, and $P$ less than 0.05 was considered to be statistically significant. In vivo tumor size compar- 
isons across different time points were compared with 2-way ANOVA (treatment and time as 2 factors), each measurement was considered independent, and the treatment $P$ value was reported. Tumor size comparison of MDA-MB-231 xenograft model at the single time point was compared using 1-sided $t$ test. Correlation analysis was performed using Spearman's rank test, and the corresponding $P$ value and correlation coefficient were reported. $P$ less than 0.05 was considered significant.

Study approval. All animal experiments were performed in accordance with protocols approved by the Institutional Animal Care and Use Committee at The University of Texas MD Anderson Cancer Center (Houston, Texas, USA).

\section{Author contributions}

$\mathrm{XX}, \mathrm{GBM}$, and HL conceived of and designed the research. XX led the experiments, and YW led the data analysis. KM, ZZ, KJJ, SY, YHT, and YL performed the experiments. LSM, CRA, GLB, and AKS provided reagents. XX, YW, GBM, and HL wrote the manuscript with input from all authors. HL supervised the whole project.

\section{Acknowledgments}

This study was supported in part by grants from the NIH (CA175486 and CA209851 to HL, CCSG grant CA016672, UH3 TR000943, R35 CA209904), a University of Texas System STARS award (to HL), the National Natural Scientific Foundation of China (81572777 to XX), a fellowship from the Gulf Coast Consortia on the Computational Cancer Biology Training Program (Cancer Prevention and Research Institute of Texas grant RP170593 to YW), and a Research Professor Award from the American Cancer Society to (AKS). We thank the MD Anderson high-performance computing core facility for computing, and LeeAnn Chastain for editorial assistance.

Address correspondence to: Han Liang, Department of Bioinformatics and Computational Biology, The University of Texas MD Anderson Cancer Center, 1400 Pressler Street, Houston, Texas 77030, USA. Phone: 713.745.9815; Email: hliang1@ mdanderson.org.
1. Bass BL. RNA editing by adenosine deaminases that act on RNA. Annu Rev Biochem. 2002;71:817-846.

2. Keegan LP, Gallo A, O'Connell MA. The many roles of an RNA editor. Nat Rev Genet. 2001;2(11):869-878.

3. Bazak L, et al. A-to-I RNA editing occurs at over a hundred million genomic sites, located in a majority of human genes. Genome Res. 2014;24(3):365-376.

4. Ramaswami G, Li JB. RADAR: a rigorously annotated database of A-to-I RNA editing. Nucleic Acids Res. 2014;42(D1):D109-DD13.

5. Ramaswami G, Lin W, Piskol R, Tan MH, Davis C, Li JB. Accurate identification of human Alu and non-Alu RNA editing sites. Nat Methods. 2012;9(6):579-581.

6. Fumagalli D, et al. Principles governing A-to-I RNA editing in the breast cancer transcriptome. Cell Rep. 2015;13(2):277-289.

7. Han L, et al. The genomic landscape and clinical relevance of A-to-I RNA editing in human cancers. Cancer Cell. 2015;28(4):515-528.

8. Paz-Yaacov N, et al. Elevated RNA editing activity is a major contributor to transcriptomic diversity in tumors. Cell Rep. 2015;13(2):267-276.

9. Chen LL, et al. Recoding RNA editing of AZIN1 predisposes to hepatocellular carcinoma. $\mathrm{Nat}$ Med. 2013;19(2):209-216.

10. Peng X, et al. A-to-I RNA editing contributes to proteomic diversity in cancer. Cancer Cell. 2018;33(5):817-828.e7.

11. Gong J, et al. LNCediting: a database for functional effects of RNA editing in lncRNAs. Nucleic Acids Res. 2017;45(D1):D79-D84.

12. Gong J, et al. Comprehensive analysis of human small RNA sequencing data provides insights into expression profiles and miRNA editing. RNA Biol. 2014;11(11):1375-1385.

13. Alon S, et al. Systematic identification of edited microRNAs in the human brain. Genome Res. 2012;22(8):1533-1540.

14. Garzon R, Calin GA, Croce CM. MicroRNAs in cancer. Annu Rev Med. 2009;60:167-179.
15. Bartel DP. MicroRNAs: genomics, biogenesis, mechanism, and function. Cell. 2004;116(2):281-297.

16. Wang Y, Liang $\mathrm{H}$. When MicroRNAs meet RNA editing in cancer: a nucleotide change can make a difference. Bioessays. 2018;40(2):1700188.

17. Choudhury Y, et al. Attenuated adenosine-toinosine editing of microRNA-376a* promotes invasiveness of glioblastoma cells. J Clin Invest. 2012;122(11):4059-4076.

18. Shoshan E, et al. Reduced adenosine-to-inosine miR-455-5p editing promotes melanoma growth and metastasis. Nat Cell Biol. 2015;17(3):311-321.

19. Cesarini V, et al. ADAR2/miR-589-3p axis controls glioblastoma cell migration/invasion. Nucleic Acids Res. 2018;46(4):2045-2059.

20. Velazquez-Torres G, et al. A-to-I miR-378a-3p editing can prevent melanoma progression via regulation of PARVA expression. Nat Commun. 2018;9(1):461.

21. Wang $Y$, et al. Systematic characterization of A-to-I RNA editing hotspots in microRNAs across human cancers. Genome Res. 2017;27(7):1112-1125.

22. Hamann J, et al. International Union of Basic and Clinical Pharmacology. XCIV. Adhesion G protein-coupled receptors. Pharmacol Rev. 2015;67(2):338-367.

23. Chiang HR, et al. Mammalian microRNAs: exper imental evaluation of novel and previously annotated genes. Genes Dev. 2010;24(10):992-1009.

24. Chen JS, et al. MicroRNA-379-5p inhibits tumor invasion and metastasis by targeting FAK/AKT signaling in hepatocellular carcinoma. Cancer Lett. 2016;375(1):73-83.

25. Coustan-Smith E, et al. New markers for minimal residual disease detection in acute lymphoblastic leukemia. Blood. 2011;117(23):6267-6276.

26. Bonardi F, Fusetti F, Deelen P, van Gosliga D, Vellenga E, Schuringa JJ. A proteomics and transcriptomics approach to identify leukemic stem cell (LSC) markers. Mol Cell Proteomics. 2013;12(3):626-637.

27. Aust G, et al. CD97: a dedifferentiation marker in human thyroid carcinomas. Cancer Res.
1997;57(9):1798-1806.

28. Aust G, et al. CD97, but not its closely related EGF-TM7 family member EMR2, is expressed on gastric, pancreatic, and esophageal carcinomas. Am J Clin Pathol. 2002;118(5):699-707.

29. Steinert M, et al. Expression and regulation of CD97 in colorectal carcinoma cell lines and tumor tissues. Am J Pathol. 2002;161(5):1657-1667.

30. Ward Y, et al. CD97 amplifies LPA receptor signaling and promotes thyroid cancer progression in a mouse model. Oncogene. 2013;32(22):2726-2738.

31. Hsiao CC, et al. The adhesion GPCR CD97/ ADGRE5 inhibits apoptosis. Int J Biochem Cell Biol. 2015;65:197-208.

32. Martin GH, et al. CD97 is a critical regulator of acute myeloid leukemia stem cell function. Blood. 2016;128(22):1077.

33. Pecot CV, et al. Tumour angiogenesis regulation by the miR-200 family. Nat Commun. 2013;4:2427.

34. Sercombe L, Veerati T, Moheimani F, Wu SY, Sood AK, Hua S. Advances and challenges of liposome assisted drug delivery. Front Pharmacol. 2015;6:286.

35. Langenhan T, Aust G, Hamann J. Sticky signaling - adhesion class $\mathrm{G}$ protein-coupled receptors take the stage. Sci Signal. 2013;6(276):re3.

36. Wobus M, Vogel B, Schmücking E, Hamann J, Aust G. N-glycosylation of CD97 within the EGF domains is crucial for epitope accessibility in normal and malignant cells as well as CD55 ligand binding. Int J Cancer. 2004;112(5):815-822.

37. Wu J, Lei L, Wang S, Gu D, Zhang J. Immunohistochemical expression and prognostic value of CD97 and its ligand CD55 in primary gallbladder carcinoma. J Biomed Biotechnol. 2012;2012:587672.

38. Safaee M, et al. Overexpression of CD97 confers an invasive phenotype in glioblastoma cells and is associated with decreased survival of glioblastoma patients. PLoS One. 2013;8(4):e62765.

39. Hamann J, Vogel B, van Schijndel GM, van Lier RA. The seven-span transmembrane receptor CD97 has a cellular ligand (CD55, DAF). J Exp 
Med. 1996;184(3):1185-1189.

40. Stacey M, et al. The epidermal growth factor-like domains of the human EMR2 receptor mediate cell attachment through chondroitin sulfate glycosaminoglycans. Blood. 2003;102(8):2916-2924.

41. Wang T, et al. CD97, an adhesion receptor on inflammatory cells, stimulates angiogenesis through binding integrin counterreceptors on endothelial cells. Blood. 2005;105(7):2836-2844.

42. Wandel E, Saalbach A, Sittig D, Gebhardt C, Aust G. Thy-1 (CD90) is an interacting partner for CD97 on activated endothelial cells. JImmunol. 2012;188(3):1442-1450.

43. Ward Y, et al. LPA receptor heterodimerizes with CD97 to amplify LPA-initiated RHO-dependent signaling and invasion in prostate cancer cells. Cancer Res. 2011;71(23):7301-7311.

44. Ozcan G, Ozpolat B, Coleman RL, Sood AK, Lopez-Berestein G. Preclinical and clinical devel- opment of siRNA-based therapeutics. Adv Drug Deliv Rev. 2015;87:108-119.

45. Cox DBT, et al. RNA editing with CRISPR-Cas13. Science. 2017;358(6366):1019-1027.

46. Rupaimoole R, Slack FJ. MicroRNA therapeutics: towards a new era for the management of cancer and other diseases. Nat Rev Drug Discov. 2017;16(3):203-222.

47. Cox DR. Regression models and life-tables. In: Kotz S, Johnson NL, eds. Breakthroughs in Statistics. Volume II. New York, New York, USA: Springer; 1992:527-541.

48. Macbeth MR, Schubert HL, Vandemark AP, Lingam AT, Hill CP, Bass BL. Inositol hexakisphosphate is bound in the ADAR2 core and required for RNA editing. Science. 2005;309(5740):1534-1539.

49. Kim D, Pertea G, Trapnell C, Pimentel H, Kelley R, Salzberg SL. TopHat2: accurate alignment of transcriptomes in the presence of inser- tions, deletions and gene fusions. Genome Biol. 2013;14(4):R36.

50. Anders S, Pyl PT, Huber W. HTSeq - a Python framework to work with high-throughput sequencing data. Bioinformatics. 2015;31(2):166-169.

51. Robinson MD, McCarthy DJ, Smyth GK. edgeR: a Bioconductor package for differential expression analysis of digital gene expression data. Bioinformatics. 2010;26(1):139-140.

52. Subramanian A, et al. Gene set enrichment analysis: a knowledge-based approach for interpreting genome-wide expression profiles. Proc Natl Acad Sci US A. 2005;102(43):15545-15550.

53. Pecot CV, et al. Therapeutic silencing of KRAS using systemically delivered siRNAs. Mol Cancer Ther. 2014;13(12):2876-2885.

54. Liu Y, et al. TP53 loss creates therapeutic vulnerability in colorectal cancer. Nature. 2015;520(7549):697-701. 